\title{
Gemcitabine: End of a Chemotherapy's Era?
}

\author{
Stephen D. Smith \\ Department of Internal Medicine, Division of Medical Oncology, University of Washington/Fred Hutchinson Cancer \\ Research Center, Seattle, WA, USA
}

Chemotherapy for relapsed lymphoma is a story of diminishing returns - of small successes but bigger setbacks, as remissions become elusive and shorter-lasting, and toxicities mount. Aggressive B- and T-cell lymphomas may manifest this pattern over a short number of years, and indolent lymphomas more gradually; but, in both settings, it is uncommon for patients to achieve durable clinical benefit with chemotherapy alone beyond the second-line setting.

The present data from Zlotnick and colleagues [1] afford a real-world perspective on the benefit of gemcitabine in this setting, providing contrast data from prospective clinical trials. Trial data, by definition, involves selection bias and imperfect translation to clinical practice. On the other hand, the homogeneity and quality of data in trials is often superior to observational data such as the present report. Thus, interpretation of this data comes with several considerations.

First, the primary endpoint of this observational study was response rate - but responses were assessed at nondefined timepoints and did not rely on imaging in $9(22 \%)$ patients. This makes interpretation of the short progression-free survival challenging. Second, actual gemcitabine treatment regimens varied, and histologies were diverse without routine assessment for MYC dysregulation; these included T-cell lymphomas with uniquely poor responses to salvage chemotherapy. Patient selection for gem-

\section{KARGER}

(C) 2019 S. Karger AG, Basel

E-Mail karger@karger.com

www.karger.com/aha citabine (as opposed to more intensive therapies, or clinical trials) may have resulted in higher-risk patients receiving it, whose outcomes with any chemotherapy may be predetermined as poor. After all, the included patients received a median of 2 prior regimens (including anthracyclines in all patients, and half failing platinum) - at a median 1.64 years after diagnosis. One patient failed gemcitabine at 12 days, a "kinetic failure" by some descriptions. Early chemotherapy failure and refractoriness to rituximab and anthracycline-containing therapy portend a poor response to salvage, and vastly inferior outcomes. The advent of safer transplantation strategies and cellular immunotherapy (chimeric antigen receptor T-cell [Car$\mathrm{T}]$ therapy), and a panoply of novel, targeted agents, has provided better options for early chemotherapy failures, ideally in the context of prospective clinical trials.

It is worth remembering that gemcitabine has definite advantages, including a large volume of supporting data across oncology, and use in lymphoma for nearly 2 decades [2]. Often relevant to relapsed lymphoma patients, gemcitabine is relatively safe in moderate kidney dysfunction or elevated transaminases, though the drug can cause worsening of hyperbilirubinemia [3,4]. Toxicities are primarily hematologic, and other than rare cases of thrombotic microangiopathy, gemcitabine has a predictable safety profile. When directly compared to inpatient salvage therapy for aggressive lymphoma, gemcitabine- 
based therapy is similar and offers outpatient administration [5]. Indirectly, it appears inclusion of platinum chemotherapy may not be required for efficacy; and nonplatinum combinations may be safer [6]. In the future, including at our center, gemcitabine is likely to remain an option for study in combination with novel agents and immunotherapies - representing a predictable, logistically feasible chemotherapy backbone.

Replacing cytotoxic chemotherapy with tumor-specific, safer, curative therapies in relapsed lymphoma remains our shared goal. These data confirm the need for discretion in selecting gemcitabine therapy for a given patient and confirm that refractory patients may benefit minimally. Nonetheless, the balance of data does not preclude gemcitabine's use in novel combinations or bridging to transplant or cellular immunotherapy. Nor should palliative use be prohibited outright - these patients may tolerate therapy well, gain a complete response, and enjoy months of clinical benefit without extensive non-hematologic toxicity. While we may all look forward to the day we may write gemcitabine off, it is too early to compose its eulogy.

\section{Disclosure Statement}

The author declares the following conflicts of interest: Research Funding from Acerta Pharma BV, Astrazeneca, Ayala (spouse), Bristol Myers Squibb (spouse), De Novo Biopharma, Genentech, Ignyta (spouse), Incyte Corporation, Merck Sharp and Dohme Corp., Pharmacyclics, Portola Pharmaceuticals, Seattle Genetics. Consultancy with Merck Sharp and Dohme Corp. Advisory Board with Astrazeneca.

\section{References}

1 Zlotnick M, Avigdor A, Ribakovsky E, Nagler A, Kedmi M. Efficacy of gemcitabine as salvage therapy for relapsed and refractory aggressive Non-Hodgkin Lymphoma. Acta Haematol. DOI: 10.1159/000495283.

2 Dumontet C, Morschhauser F, Solal-Celigny P, Bouafia F, Bourgeois E, Thieblemont C, et al. Gemcitabine as a single agent in the treatment of relapsed or refractory low-grade nonHodgkin's lymphoma. Br J Haematol. 2001 Jun;113(3):772-8.
3 Delaloge S, Llombart A, Di Palma M, Tourani JM, Turpin F, Ni L, et al. Gemcitabine in patients with solid tumors and renal impairment: a pharmacokinetic phase I study. Am J Clin Oncol. 2004 Jun;27(3):289-93.

4 Venook AP, Egorin MJ, Rosner GL, Hollis D, Mani S, Hawkins M, et al. Phase I and pharmacokinetic trial of gemcitabine in patients with hepatic or renal dysfunction: Cancer and Leukemia Group B 9565. J Clin Oncol. 2000 Jul;18(14):2780-7.
5 Crump M, Kuruvilla J, Couban S, MacDonald DA, Kukreti V, Kouroukis CT, et al. Randomized comparison of gemcitabine, dexamethasone, and cisplatin versus dexamethasone, $\mathrm{cy}$ tarabine, and cisplatin chemotherapy before autologous stem-cell transplantation for relapsed and refractory aggressive lymphomas: NCIC-CTG LY.12. J Clin Oncol. 2014 Nov; 32(31):3490-6.

6 Müller-Beissenhirtz H, Kasper C, Nückel H, Dührsen U. Gemcitabine, vinorelbine and prednisone for refractory or relapsed aggressive lymphoma, results of a phase II single center study. Ann Hematol. 2005 Nov;84(12): 796-801. 